\title{
Graph Representation of Road and Traffic for Autonomous Driving
}

\author{
Jianglin Qiao $^{1}$, Dongmo Zhang $^{1}$, and Dave de Jonge ${ }^{1,2}$ \\ 1 University of Western Sydney, Australia, \\ 19469397@student. westernsydney.edu.au, \\ \{d.zhang, d.dejonge\}@westernsydney.edu.au \\ 2 IIIA-CSIC, Spain \\ davedejongediiia.csic.es
}

\begin{abstract}
Autonomous driving has the potential to radically change the way vehicles interact each other. This paper aims to develop a formal method to model high level interaction between autonomous vehicles. We introduce a concept of road graph to represent complex road situations such as intersections, road merging, unmarked roads, and traffic hazards. We then extend the concept to further represent status of vehicles, dynamics of traffic and protocols of traffic control. Specifically, we formalise two categories of traffic control protocols, time-based protocols and priority-based protocols.
\end{abstract}

\section{Introduction}

Over the last decade, research on autonomous vehicles (AVs) has made revolutionary progress, which brings us hope of safer, more convenient and efficient means of transportation [2,9]. A great number of tech giants and research institutes alone with the major automakers are striving for the developments of new technologies for autonomous driving with the ultimate target that all vehicles eventually become fully autonomous without human intervention at their highest automation level $[4,8]$.

An autonomous vehicle system is an integration of many technologies, including computer vision, graphical processing, navigation, sensor technologies and so on. Most significantly, the recent advance of machine learning technologies enables a self-driving car to learn to drive in any complex road situations with millions of accumulated driving hours, which are way higher than any experienced human driver can reach. However, driving is not a purely technical job but involves complicated social activities, which could be hard to learn from experience. For instance, if two cars meet in a narrow road or a long bridge on which only one car can go through, how do the cars decide which one should reverse to give way to the other? Many of such a situation requires direct interaction among vehicles, vehicles and infrastructures, or vehicles and authorities $[3,5]$. Such demands push the research on AVs to a different direction from machine learning with regards to communication, negotiation and cooperation among autonomous vehicles. Unfortunately the studies along, this direction is far from adequate.

Existing research on vehicle to vehicle (V2V) allows exchange of information collected from local sensors to achieve a form of 'collaborative awareness' [1,7]. However, the information exchanged between vehicles are normally signal data or messages. Such low level communication cannot achieve high-level interaction for the purpose of automated negotiation, cooperative maneuvering, collaborative auto-piloting among autonomous vehicles [6]. 
The primary goal of our research is to establish a high-level communication protocol for autonomous vehicles, which can solve various traffic conditions through negotiation between vehicles. Herein, we propose the road graph; a novel concept for representing roads and traffic which includes fully autonomous vehicles, as the basis of our research. With this road graph model we describe various traffic-related elements, such as traffic flow, traffic-control protocols, vehicle information and vehicle management processes. It should be mentioned that at this point our work is only for theoretical research. Its purpose is to develop an abstract model that allows self-driving vehicles to understand roads and traffic, so as to pave the way for more advanced vehicle negotiation. We leave it as future work to develop models that are closer to real-world traffic.

The structure of this paper is as follows. In Section 2 we introduce the representation of roads, vehicle and traffic setting, and traffic state and traffic flow. In section 3, we give traffic control protocols in detailed. In Section 4 we summarise the paper with conclusions and future research directions.

\section{Traffic representation}

To allow autonomous vehicles to reason about complicated traffic situations, we introduce a formal method to represent roads, traffic flows and traffic control protocols. As a generic assumption of this work and a way of abstraction, time occurs at distinct, separate "points in time", throughout each non-zero region of time ("time period"), represented by natural numbers $T=\{1,2,3, \cdots\}$.

\subsection{Road graph}

In order to specify any complicated road, we divide a road into a number of blocks or road segments. Each block of a road allows one car to travel at each time ${ }^{3}$, represented as a vertex in a graph. Directed edges in a graph represent connections and travel directions between road blocks. If two vertices have no edge to link them, no vehicle can travel directly between these two blocks. In addition, we assume that each road contain a number of entries and a number of exits. For each entry, it must have at least one out-going edge, and for each exit, it must have at least one incoming edge. Formally we have the following definition.

Definition $1 A$ road graph $G$ is a tuple $\left(B, \mathcal{E}, B_{n}, B_{x}\right)$, where:

- $B$ is a non-empty and finite set of blocks;

- $\mathcal{E} \subseteq B \times B$ is a set of arcs. An arc $\left(b, b^{\prime}\right) \in \mathcal{E}$ refers to a connection and travel direction from block $b$ to block $b^{\prime}$;

- $B_{n} \subseteq B$ is a set of blocks to represent the road entries;

- $B_{x} \subseteq B$ is a set of blocks to represent the road exits.

Figure 1 shows a simple road graph representing a typical two-way two-lane road. The vertices $b_{1}, \cdots, b_{20}$ represent the segments of the road while the arcs indicate the traffic flows that are allowed from segments to segments. For instance, $b_{1}, b_{2}, b_{3}, b_{4}, b_{5}$ are the blocks of the left most lane. The arc from $b_{2}$ to $b_{8}$ means a vehicle on block $b_{2}$ can change lane to block $b_{8}$ but is not allowed to shift to block $b_{7}$. Blocks $b_{1}, b_{6}$, $b_{11}$ and $b_{16}$ are entries of the road, and blocks $b_{5}, b_{10}, b_{15}$ and $b_{20}$ are exits of the road.

\footnotetext{
${ }^{3}$ We will use discrete time to represent traffic flows thus a time point represents a period of time.
} 

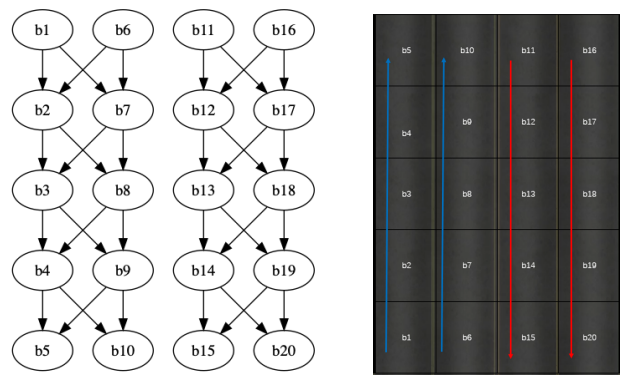

Fig. 1. A road graph representing a two-way two-lane road

As an abstraction of roads, a road graph can represent more complicated road situations and configurations, such as multi-way junctions, roundabout, no-through road and U-turns. The following example shows a representation of a typical four-way intersection.

Example 1 Figure 2 shows an example of road graph for a four-way intersection which will be used as running example of the paper. Each direction has one lane. Note that the road graph indicates that a vehicle turning right is not allowed to travel in diagonal inside the intersection. For instance, a vehicle at $b_{2}$ turning to $b_{19}$ must travel via $b_{3}$, $b_{4}$ and $b_{10}$ rather than a sharp turn from $b_{3}$ to $b_{10}$. This is by no means a restriction of road graph representation but reflects an actual setting of the road.

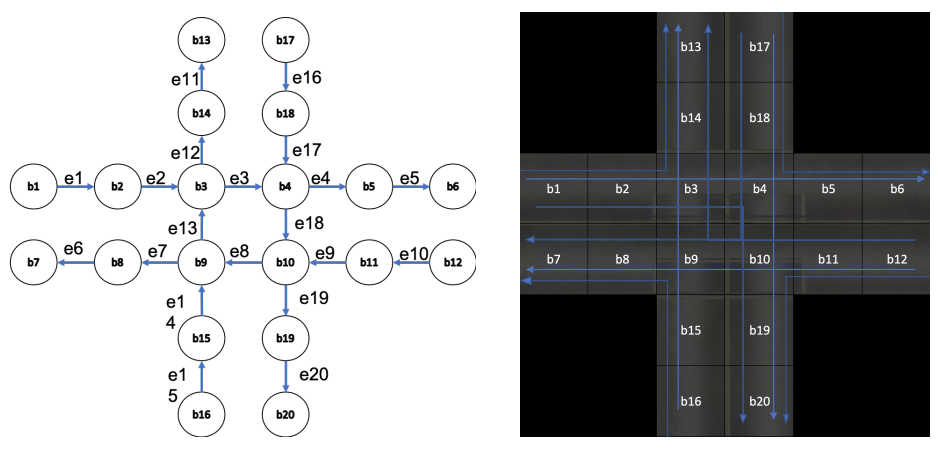

Fig. 2. A road graph for four-way intersection

Given a road graph, a road path can be easily defined as path in terms of the standard graph theory terminology. Formally we have the following definition:

Given a road graph $\left(B, \mathcal{E}, B_{n}, B_{x}\right)$, a path $\rho$ is a sequence $b_{0} \stackrel{e_{1}}{\rightarrow} b_{1} \stackrel{e_{2}}{\rightarrow} b_{2} \ldots \stackrel{e_{m}}{\rightarrow}$ $b_{m}$, where

- $b_{i} \in B$ for all $0 \leq i \leq m$

- $e_{i}=\left(b_{i-1}, b_{i}\right) \in \mathcal{E}$ for all $0<i \leq m$

- $b_{i} \neq b_{j}$ for any $i \neq j$

$\rho$ is called a complete path if $b_{0} \in B_{n}$ and $b_{m} \in B_{x}$. We use $\hat{\rho}$ to denote the start block of the path and $\check{\rho}$ the end block of the path, i.e., $\hat{\rho}=b_{0}$ and $\check{\rho}=b_{m}$. 


\subsection{Vehicles and traffic settings}

Vehicles are road users. We assume that all vehicles are fully autonomous, which means that decision-making of each vehicle is not centralised but by the vehicle itself, no matter they driven by human or computers. We also assume that each vehicle has a designated complete path, which specified its entry block, exit block and intended travel path. In the context of automated negotiation between autonomous vehicles, these pieces of information are initial settings of a vehicle before it enters a road. They are negotiable when it travels on the road. In addition, we also assume each vehicle has a designated time point to enter the road. Formally, we specify the information of vehicles with the following concept:

Definition 2 Given a road graph $G=\left(B, \mathcal{E}, B_{n}, B_{x}\right)$ and a set $\mathcal{V}$ of possible vehicles, the vehicle information, $\mathcal{I}$, on $G$ is represented by a tuple $(\mu, \sigma, \eta, \mathcal{P})$ where

- $\mu: \mathcal{V} \rightarrow B_{n}$ is a function that maps each vehicle to a road entry;

- $\sigma: \mathcal{V} \rightarrow B_{x}$ is a function that maps each vehicle to a road exit;

- $\eta: \mathcal{V} \rightarrow T$ is a function that maps each vehicle to a time point indicating the time the vehicle is expected to enter the road;

$-\mathcal{P}: \mathcal{V} \rightarrow P$ is a function that maps each vehicle to a complete path such that for each vehicle $v \in \mathcal{V}, \hat{\mathcal{P}} \hat{(v)}=\mu(v)$ and $\mathcal{P}(v)=\sigma(v)$.

In the rest of the paper, we call $(G, \mathcal{V}, \mathcal{I})$ a traffic setting.

\subsection{Traffic states and traffic flows}

Traffic means that vehicles move on a road. A snapshot of traffic on a road can be viewed as a set of vehicles that currently on the road and the positions they occupy. As we mentioned before, we assume that each block can only contain one vehicle at each time point. Therefore vehicles' location can be represented with an injective function from a vehicle to a block of the road. Formally we introduce the following concept:

Definition 3 Given a traffic setting $(G, \mathcal{V}, \mathcal{I}), a$ traffic state with respect to the traffic setting is a pair $(V, \tau)$ where

$-V \subseteq \mathcal{V}$, indicating the vehicles that are currently on the road;

- $\tau: V \rightarrow B$ is an injective function that maps each vehicle to a block of the road. In other words, for any $v, v^{\prime} \in V, \tau(v)=\tau\left(v^{\prime}\right)$ implies $v=v^{\prime}$.

A traffic state represents a snapshot of a traffic flow, thus, is a static view of traffic. However, traffic is dynamic. In order to model a flow of traffic, we define traffic on a road as a set of traffic states in time sequence:

Definition 4 Let $(G, \mathcal{V}, \mathcal{I})$ be a traffic setting. A traffic flow $\mathcal{F}=\left\langle\left(V_{t}, \tau_{t}\right)\right\rangle_{t \in T}$ is a temporal sequence of traffic states such that for each time point $t \in T$,

1. $v \in V_{t+1} \backslash V_{t}$ implies $\tau_{t+1}(v)=\mu(v)$, i.e., a vehicle must enter the road from its specified entry.

2. for each $v \in V_{t}$, exactly one of the following conditions holds

(a) $\tau_{t}(v)=\tau_{t+1}(v)$

(b) $\left(\tau_{t}(v), \tau_{t+1}(v)\right) \in \mathcal{E}$

(c) $\tau_{t}(v)=\sigma(v)$ and $v \notin V_{t+1}$

3. for any $v, v^{\prime} \in V_{t}$ such that $v \neq v^{\prime}$ and $\tau_{t}(v)=\tau_{t+1}\left(v^{\prime}\right), \tau_{t+1}(v) \neq \tau_{t}\left(v^{\prime}\right)$. 


\section{Traffic control protocols}

Traffic must be controlled to ensure road safety and efficiency. The way of traffic control is to instruct vehicles taking appropriate measures or actions in order to avoid collisions or delays. Complex operational procedures, rules and laws, and physical equipment (such as signs, markings, and lights) have been used in real-world traffic control systems. The most common traffic control devices and methods are traffic lights, stop signs, roundabouts and other facilities. Despite of the significant differences between different traffic control methods and systems, the mechanisms of all traffic control methods can be categorised into two fundamental traffic control protocols: time-based traffic control and priority-based traffic control.

\subsection{Time-based traffic control protocols}

The time-based traffic control utilises protocols that control traffic by restricting accessibility or impassability of roads in different time periods. With the road graph representation, we can simply define a time-based traffic protocol as a temporal sequence of arc groups, indicating which road segments are accessible at each time. A typical application of time-based traffic control protocols is traffic light systems.

Definition 5 Given a road graph $G=\left(B, \mathcal{E}, B_{n}, B_{x}\right)$. A time-based traffic control protocol $\alpha: T \rightarrow 2^{\mathcal{E}}$ is a function from each time point to a subset of arcs. Furthermore, a traffic flow $\mathcal{F}=\left\langle\left(V_{t}, \tau_{t}\right)\right\rangle_{t \in T}$ is said to be complied with a time-based protocol $\alpha$ if for any time point $t$ and any vehicle $v$, if $v \in V_{t}, \tau_{t}(v) \neq \sigma(v)$ and $\tau_{t}(v) \neq \tau_{t+1}(v)$, then $\left(\tau_{t}(v), \tau_{t+1}(v)\right) \in \alpha(t)$.

Intuitively, a time-based traffic control protocol specifies which segment of road can go through at each time point. In other words, for each time point $t$, all the arcs in $\alpha(t)$ are passible (green light), while all the arcs in $\mathcal{E} \backslash \alpha(t)$ are impassible (red light). When a traffic control protocol is enforced on a road, traffic is shaped to form specific patterns of traffic flow. A traffic flow is complied with a time-based protocol means any vehicle must travel at green lights. The following example shows a representation of traffic in a four-way intersection when time-based protocol is enforced.

Example 2 Consider a road graph for a four-way intersection in Example1, and a time-based traffic control protocol $\alpha$ on the road graph as follows:

$$
\alpha(t)= \begin{cases}E_{g} \cup\left\{e_{2}, e_{3}, e_{9}, e_{8}\right\}, & \text { if } 0 \leq t \bmod l<1_{1} \\ E_{g} \cup\left\{e_{2}, e_{3}, e_{18}, e_{14}\right\}, & \text { if } l_{1} \leq t \bmod l<l_{2} \\ E_{g} \cup\left\{e_{14}, e_{13}, e_{17}, e_{18}\right\}, & \text { if } l_{2} \leq t \bmod l<l_{3} \\ E_{g} \cup\left\{e_{9}, e_{8}, e_{13}, e_{17}\right\}, & \text { if } l_{3} \leq t \bmod l<l\end{cases}
$$

where $0<l_{1}<l_{2}<l_{3}<l$ and $E_{g}=\left\{e_{1}, e_{4}, e_{5}, e_{6}, e_{7}, e_{10}, e_{11}, e_{12}, e_{15}, e_{16}, e_{19}, e_{20}\right\}$. $t$ mod l means " $t$ modulo $l$ ".

The protocol specifies four time intervals in each period of length $l:\left[0, l_{1}\right),\left[l_{1}, l_{2}\right)$, $\left[l_{2}, l_{3}\right)$ and $\left[l_{3}, l\right)$. The first time interval allows vehicles from east or west to travel straight or take a left turn. The second interval allows traffic from west to take right turn and traffic from south to take left turn. The other two intervals are similar for traffic from other directions. 


\subsection{Priority-based traffic control protocol}

A priority-based protocol controls traffic based on preset priorities of roads at each road junction. For instance, in a left driving country, vehicles give way to the traffic on right whenever they are approaching an intersection, which means that the road on right has higher priority than the road a vehicle travels on. With the help of graph representation of roads, we can formalise a priority-based traffic control protocol as follows:

Definition 6 Given a road graph $G=\left(B, \mathcal{E}, B_{n}, B_{x}\right)$. A priority-based protocol $\beta$ : $\mathcal{E} \rightarrow 2^{\mathcal{E}}$ is a function from each arc of the road to a subset of the arcs $\mathcal{E}$ such that

1. for any $\left(b_{1}, b_{2}\right) \in \mathcal{E}$, if $\left(b_{1}^{\prime}, b_{2}^{\prime}\right) \in \beta\left(b_{1}, b_{2}\right)$, then $b_{2}=b_{2}^{\prime}$;

2. if $e^{\prime} \in \beta(e), e \notin \beta\left(e^{\prime}\right)$.

To understand the conditions of the definition, the first condition means that an arc gives priority to another arc only if they meet at the same block. The second condition means that two vehicles on different roads do not give way to each other. Similar to time-based traffic control protocols, we can also define whether a traffic flow is complied with a priority-based traffic control protocol.

Definition 7 Let $(G, \mathcal{V}, \mathcal{I})$ be a traffic setting. A traffic flow $\mathcal{F}=\left\langle\left(V_{t}, \tau_{t}\right)\right\rangle_{t \in T}$ is said to be complied with a priority-based protocol $\beta$ if for any $t \in T$ and any $v, v^{\prime} \in V_{t}$, such that $v \neq v^{\prime}, \tau_{t}(v) \neq \tau_{t+1}(v)$ implies $\left(\tau_{t}\left(v^{\prime}\right), \tau_{t+1}(v)\right) \notin \beta\left(\tau_{t}(v), \tau_{t+1}(v)\right)$ unless $\left(\tau_{t}\left(v^{\prime}\right), \tau_{t+1}(v)\right) \notin \mathcal{P}(v)$.

It means that a vehicle does not have to give way to another vehicle only if the road that the other vehicle travels on does not have a higher priority or the other vehicle does not travel into the same block.

Example 3 Figure 3 shows a road graph representing a T-junction. Imagine there is a stop sign in block $b_{15}$. Then all vehicles entering $b_{9}$ via $e_{14}$ must stop at the stop sign and observe the coming vehicles towards $b_{9}$ from other roads. Assume that we enforce the following priority-based traffic control protocol $\beta$ at this T-junction:

- $\beta(e)=\emptyset$, where $e \in\left\{e_{1}, e_{3}, e_{4}, e_{5}, e_{6}, e_{7}, e_{8}, e_{10}, e_{11}, e_{13}, e_{14}, e_{15}, e_{16}\right\}$.

- $\beta\left(e_{2}\right)=\left\{e_{13}\right\} ; \beta\left(e_{12}\right)=\left\{e_{8}\right\} ; \beta\left(e_{9}\right)=\left\{e_{14}\right\} ;$

Let $\mathcal{F}(t)=\left(V_{t}, \tau_{t}\right)$ be a traffic state at time $t$ where $V_{t}=\left\{v_{1}, v_{2}\right\}, \tau_{t}\left(v_{1}\right)=b_{10}$ and $\tau_{t}\left(v_{2}\right)=b_{15}$. Assume that both vehicles $v_{1}$ and $v_{2}$ are travelling towards block $b_{9}$. Since $e_{8}$ has a higher priority than $e_{14}$, only vehicle $v_{1}$ can go through but $v_{2}$ must stay in block $b_{15}$. If $\left.\mathcal{F}(t+1)\right)=\left(V_{t+1}, \tau_{t+1}\right)$ represents the next state, we then have $\tau_{t+1}\left(v_{1}\right)=b_{9}$ and $\tau_{t+1}\left(v_{2}\right)=b_{15}$.

\section{Conclusion and future work}

In this paper, we proposed a way to represent traffic roads, traffic flow, and control protocol at first. And then we introduce a process to generate a traffic flow within traffic control protocols, which are time-based protocol and priority-based protocol. Autonomous vehicle will determine the future of road transportation systems. These 


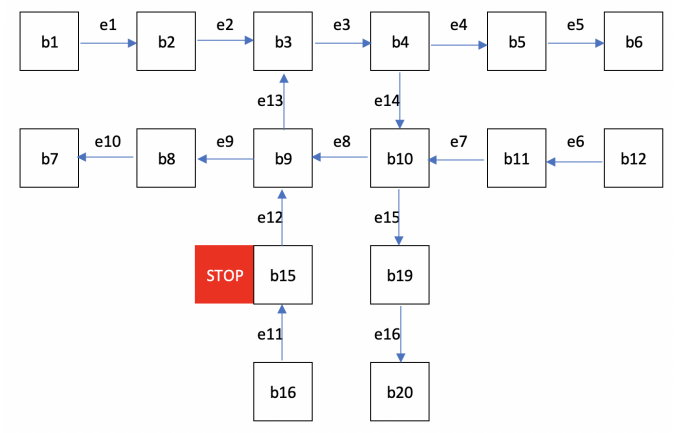

Fig. 3. An example of priority-based protocol on a T-junction

technologies aim to improve mobility, safety, comfort, and fuel consumption while reducing emissions. However, the extent of this improvement is unknown. Although the impact on the driving environment has extensively analysed in the literature, more comprehensive studies are needed. Our research can not only describe a single type of traffic situation but also cover different traffic situations. However, this article is just the basis for our research. We will pave the way for future vehicle negotiations by indicating roads, traffic flow, and traffic control protocols.

\section{References}

1. Azimi, S., Bhatia, G., Rajkumar, R., Mudalige, P.: Reliable intersection protocols using vehicular networks. In: 2013 ACM/IEEE International Conference on Cyber-Physical Systems (ICCPS). pp. 1-10, 2013.

2. Banerjee, T., Bose, S., Chakraborty, A., Samadder, T., Kumar, B., Rana, T.K.: Self driving cars: A peep into the future. In: 2017 th Annual Industrial Automation and Electromechanical Engineering Conference (IEMECON). pp. 33-38, 2017.

3. Cui, S., Seibold, B., Stern, R., Work, D.B.: Stabilizing traffic flow via a single autonomous vehicle: Possibilities and limitations. In: 2017 IEEE Intelligent Vehicles Symposium (IV). pp. 1336-1341, 2017.

4. Dikmen, M., Burns, C.M.: Autonomous driving in the real world: Experiences with tesla autopilot and summon. In: Proceedings of the 8th International Conference on Automotive User Interfaces and Interactive Vehicular Applications (AutomotiveUI). pp. 225-228, 2016.

5. Gruel, W., Stanford, J.M.: Assessing the long-term effects of autonomous vehicles: A speculative approach. Transportation Research Procedia 13, 18 - 29 (2016).

6. Hobert, L., Festag, A., Llatser, I., Altomare, L., Visintainer, F., Kovacs, A.: Enhancements of V2X communication in support of cooperative autonomous driving. IEEE Communications Magazine 53(12), 64-70, 2015.

7. Liu, C., Lin, C.W., Shiraishi, S., Tomizuka, M.: Improving efficiency of autonomous vehicles by V2V communication. In: 2018 Annual American Control Conference (ACC), pp. 47784783, 2018.

8. Ross, P.E.: Robot, you can drive my car. IEEE Spectrum 51(6), 60-90, 2014.

9. Thorpe, C., Herbert, M., Kanade, T., Shafer, S.: Toward autonomous driving: the CMU Navlab. I. Perception. IEEE Expert 6(4), 31-42, 1991. 